\title{
Importância do método de gota espessa de sangue no diagnóstico de filárias simpátricas no Amazonas, Brasil
}

\author{
Jansen F. MEDEIROS ${ }^{1}$, Felipe Arley Costa PESSOA², Marilaine MARTINS ${ }^{3}$
}

\section{RESUMO}

Esse trabalho relata os métodos de diagnósticos para filárias humanas no sangue, referindo-se a importância do método de gota espessa em relação aos métodos de filtração em membrana de policarbonato e Knott na diferenciação das espécies de microfilárias, principalmente em áreas de ocorrência de mais de uma espécie, como em regióes do Amazonas. Lâminas com microfilárias de Mansonella ozzardi foram montadas e fotografadas pelos diferentes métodos de diagnósticos. O método da gota espessa de sangue é o mais confiável, pois permite visualizar com nitidez o espaço cefálico e caudal, disposiçáo dos núcleos caudais e formato da cauda, que são características morfológicas que diferenciam as espécies de microfilárias sanguíneas que ocorrem no Amazonas.

PaLAVRAS - CHAVE: Filariose, Métodos de diagnósticos, Mansonella ozzardi, Mansonella perstans.

\section{The importance of thick blood film method for sympatric filarial diagnosis in Amazon basin}

\section{ABSTRACT}

In this work, the visual diagnostic methods for human filarias in the blood are evaluated. The thick blood film method is more faithful to identify the microfilariae species if compared to policarbonate membrane filtration and Knott methods, especially in areas where more than one species occur, as in Amazon basin. Slides with Mansonella ozzardi microfilariae were mounted and photographed by different diagnosis methods. The thick blood film method is much easier to see the diagnostic morphological characters as cephalic and caudal space, pattern of nucleation of the tail and tail shape that can separate the blood Amazonian microfilariae species.

KEYWORDS: Filariasis, Diagnostic methods, Mansonella ozzardi, Mansonella perstans.

1 Instituto Nacional de Pesquisas da Amazônia/Universidade do Estado do Amazonas, CP. 478, 69011-970, Manaus, AM; E-mail: jmedeiro@gmail.com

2 Centro de Pesquisa Leônidas e Maria Deane, Fiocruz-Amazônia; E-mail: facpessoa@amazonia.fiocruz.br

${ }^{3}$ Fundação de Medicina Tropical do Amazonas/Universidade do Estado do Amazonas, Manaus, AM; E-mail: martins.marilaine@gmail.com 
A mansonelose é uma filariose causada por Mansonella ozzardi (Manson, 1897) (Nematoda, Onchocercidae) e por M. perstans (Manson, 1891) (Nematoda, Onchocercidae). No Brasil possui uma ampla distribuição entre as populaçôes ribeirinhas e indígenas no Amazonas, e registro em Roraima e Mato Grosso. A M. ozzardi foi registrada a partir da década de 1940 (Deane 1949), já a $M$. perstans foi encontrada recentemente entre populaçôes indígenas do Alto Rio Negro (Medeiros \& Py-Daniel 2009). Uma das questôes preocupantes é a ocorrência concomitante dessas duas filárias em algumas regiôes do Amazonas, tornando-se imprescindível um diagnóstico correto devido às diferenças de tratamento. Os métodos mais utilizados no diagnóstico se baseiam na visualizaçấo das microfilárias no sangue periférico destacando-se a gota espessa, gota fresca e ainda os métodos de concentraçáo, denominadas assim porque utilizam um maior volume de sangue: concentração de Knott (Knott 1939) e filtração em membrana de policarbonato (Bell 1967). Para a realização desta nota científica, nos propomos testar a viabilidade de visualização de estruturas diagnósticas de microfilárias. Lâminas com microfilárias de $M$. ozzardi foram montadas e fotografadas pelos diferentes métodos diagnósticos. Observamos que os métodos de concentração sem a preparação da gota espessa em área de infecção mista podem induzir a dúvida no diagnóstico específico, devido à dificuldade de visualizar as principais estruturas que identificam as espécies: disposição dos núcleos, tamanho e largura do espaço cefálico e caudal e formato da cauda (Figuras $1 \mathrm{a}-\mathrm{b}$ ). A técnica de Knott consiste em diluir $1 \mathrm{~mL}$ de sangue venoso em $9 \mathrm{~mL}$ de formalina a $2 \%$, que posteriormente é centrifugado e o sedimento é removido para preparação da gota espessa. $\mathrm{O}$ fato das microfilárias ficarem misturadas ao sedimento viscoso (Figuras 1c - d) dificulta a identificação em microscópio (Rocha \& Fontes 2000). A filtração em membrana consiste em filtrar o sangue diluído em solução tamponada através de membrana de policarbonato (poros 3 ou $5 \mu \mathrm{m}$ de diâmetro) que permite a passagem de hemácias, mas retém as microfilárias, como também outros elementos que por sua vez dificultam a identificação das microfilárias (Figuras 1e - f). Embora os métodos de concentração sejam mais sensíveis, principalmente em indivíduos com baixa microfilaremia e utilizados frequentemente como controle de cura para filariose sanguínea (Dreyer \& Norôes 1997), não recomendamos sua utilização de forma isolada em levantamentos preliminares de filarioses no Amazonas. Enfatizamos que no Amazonas, o método da gota espessa de sangue ainda é o mais confiável e de baixo custo, pois permite visualizar com nitidez algumas características, como comprimento das microfilárias, tamanho e largura do espaço cefálico e caudal, formato da cauda e disposição dos núcleos caudais, que diferenciam as espécies de microfilárias sanguíneas, e assim administrar de forma correta o tratamento. Também reforçamos que esse método é o mais apropriado para a identificação de novas espécies, já que os outros métodos mencionados (filtragem em membrana e Knott) comprometem morfologicamente as microfilárias.

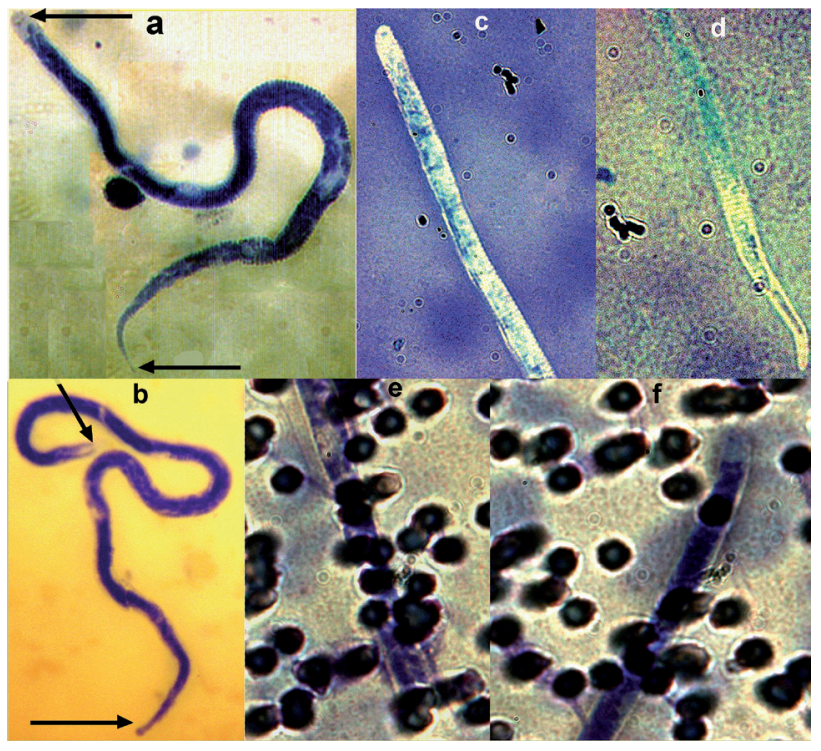

Figura 1 - Microfilárias em diferentes métodos de diagnóstico. a - b - M. ozzardi e M. perstans, respectivamente - método de gota espessa com estruturas de fácil visualização (tamanho e largura do espaço cefálico e caudal e formato da cauda, setas apontando para essas estruturas); c - d - Extremidade anterior e posterior de M. ozzardi, respectivamente - método de Knott; e - $f$ - Extremidade anterior e posterior de M. ozzardi, respectivamente - método de filtro de membrana.

Figuras a, c, d, e, f: Microfilárias de Mansonella ozzardi coletadas em pacientes do município de Coari, AM: foto Medeiros \& Pessoa.

Figura b: fonte Medeiros \& Py-Daniel (2009).

\section{BIBLIOGRAFIA CITADA}

Deane, M.P. 1949. About the incidence of human filariae in Manaus, Amazonas States. Revista do SESP, 2: 849-858 (in Portuguese, with abstract in English).

Bell, D. 1967. Membrane filters and microfilarial surveys on day blood. Transactions of the Royal Society of Tropical Medicine and Hygiene, 61: 220-223.

Dreyer, G.; Norôes, J. 1997. Dietilcarbamazine in the treatment of bancroftian filariasis. Revista da Sociedade Brasileira de Medicina Tropical, 30(3): 229-240 (in Portuguese, with abstract in English).

Knott, J.A. 1939. Method for making microfilarial surveys on day blood. Transactions of the Royal Society of Tropical Medicine and Hygiene, 32: 191-196.

Medeiros, J.F.; Py-Daniel, V. 2009. Mansoneliasis. In: Marcondes, C.B. (Ed). Diseases transmited and caused by arthropods. Atheneu Press. p. 277-282 (in Portuguese).

Rocha, E.M.M.; Fontes, R. 2000. Diagnosis of bancroftian filariasis. Revista de Patologia Tropical, 29(2): 61-73 (in Portuguese, with abstract in English).

Recebido em 08/12/2009

Aceito em 18/05/2010 